\title{
Precision Theory for Precision Measurements: Tests of the Standard Model via Parity-Violating Electron-Proton and Møller Scattering
}

\author{
Aleksandrs Aleksejevs \\ Memorial University of Newfoundland \\ Corner Brook, Newfoundland, Canada \\ E-mail:aaleksejevs@swgc.mun.ca
}

\section{Svetlana Barkanova ${ }^{1}$}

Acadia University

Wolfville, Nova Scotia, Canada

E-mail: svetlana.barkanova@acadiau.ca

\section{Alexander Ilyichev}

National Center of Particle and High Energy Physics

Minsk, Belarus

E-mail: ily@hep.by

\section{Vladimir Zykunov}

Belarusian State University of Transport

Gomel, Belarus

E-mail: vladimir.zykunov@cern.ch

As experimental techniques continue to be developed and improved, they will require more precise contributions from theory. The indirect tests of the Standard Model via high-precision measurements like $\mathrm{Q}_{\text {Weak }}$ and the $11 \mathrm{GeV}$ Møller scattering planned at JLab will demand a complete theoretical evaluation of the next-to-leading order and higher effects in electroweak interactions done with unprecedented precision. We show what kind of theoretical support our group can provide to the above, and other experiments, with the new computational methods we have developed. Some of the key features of our approach, including our method for dealing with many-body effects in $e-p$ scattering and our treatment of Hard Photon Bremsstrahlung, are discussed. 


\section{Introduction}

The Standard Model introduces an asymmetry between left and right-handed particles and predicts parity violating interference between the weak and electromagnetic forces. These interference effects are very small but have been clearly detected and can give us access to parameters like the weak charges of electrons and nucleons. Although the evidence for new physics through the indirect tests of the Standard Model via high-precision measurements can be model-dependent, low-energy electroweak measurements are an essential compliment to the LHC program. The inclusion of radiative corrections is an indispensable part of any modern experiment, but experiments like the future $11 \mathrm{GeV}$ Møller [1] and current $Q_{\text {Weak }}$ [2] at Jefferson Lab (JLab) will require next-to-leading order (NLO) electroweak radiative corrections (EWC) calculated with an unprecedented completeness and accuracy.

\section{Key features of our approach}

Computer packages such as FeynArts, FormCalc, LoopTools and Form have created the possibility to both handle the huge volume of work reasonably quickly and to avoid the rapid error accumulation often unavoidable with purely numerical methods. In general, our results can be presented in both numerical and analytical form. For $e$-e scattering, it is possible to present our results as compact analytical, yet sufficiently accurate, expressions obtained using asymptotic approximations. Unfortunately, in the case of $e-p$ scattering, the expressions are made very cumbersome by hadronic effects, so we are providing numerical data only. For lepton-proton scattering, we do the exact calculation of the model-independent corrections to the lepton current, and perform a model-dependent evaluation of hadronic current corrections, box graph and self energy contributions, and electromagnetic radiation of the charged particles (soft and hard photon bremsstrahlung). For the model-independent lepton current corrections, we can consider EWC up to the second, next-to-next-to-leading-order.

\section{Our predictions for the $11 \mathrm{GeV}^{2}$ Møller experiment}

Møller scattering measurements are a powerful probe of new physics effects. The stated precision goal of the new Møller experiment at JLab [1], which will measure the parity-violating $(\mathrm{PV})$ scattering asymmetry in the process $\mathrm{e}^{-} \mathrm{e}^{-} \rightarrow \mathrm{e}^{-}$ $\mathrm{e}^{-}(\gamma)$ at $11 \mathrm{GeV}$, is significantly more ambitious than that of its predecessor E-158, so very precise theoretical input for this measurement will be crucial. In spite of earlier theoretical effort dedicated to calculations of EWC (see refs. in [3]), we believe that a new level of accuracy is required

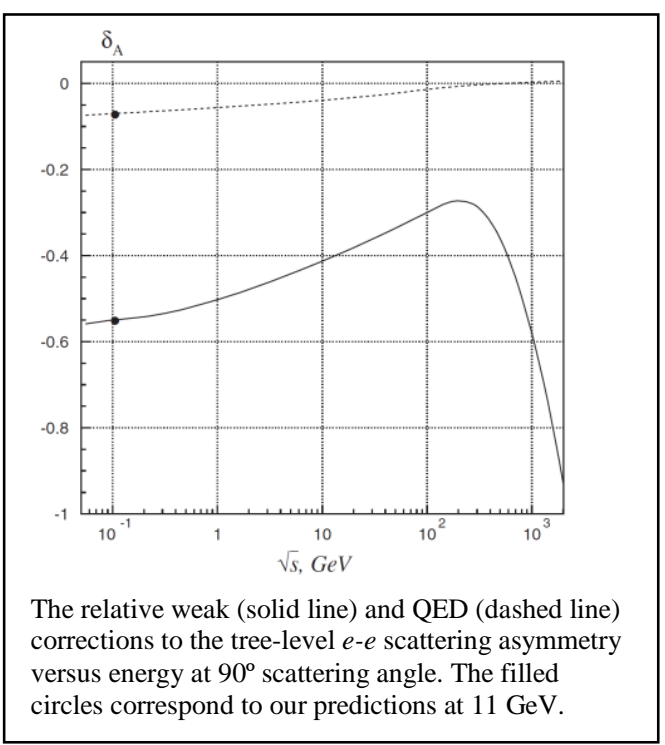


for the next-generation, high-precision experiments. Using two different renormalization conditions, we perform updated and detailed calculations of the complete one-loop set of EWC to parity-violating $e$-e scattering asymmetry at low energies relevant to the future JLab Møller experiment. Although contributions from some of the self-energy and vertex diagrams calculated in the two approaches can differ significantly, our full gauge-invariant set still guarantees that the total relative weak corrections are in excellent agreement for the two methods of calculation. We find that at certain kinematic conditions EWC can reduce the crosssection asymmetry up to $\sim 70 \%$, and they strongly depend on the experimental cuts. A detailed analysis of the various contributions with the results presented in both numerical and analytical form is given in [3].

\section{Our predictions for the $Q_{W e a k}$ experiment}

The measurement of the proton's weak charge with $\sim 4 \%$ combined statistical and systematic errors, soon to be completed by the $Q_{\text {Weak }}$ collaboration at JLab [2], may have extremely interesting physical implications. Using the FeynArts, FormCalc, LoopTools and Form packages as the base languages, we calculate the $e-p$ scattering amplitudes and differential cross sections first analytically and then numerically. To include a complete set of one-loop EWC, we take into account boson self-energies, corrections to vertex functions, two-bosonexchange diagrams, and the contributions of soft and hard bremsstrahlung. The inclusion of the Dirac and Pauli form-factor type coupling in the boson-nucleon interaction using the multipole expansion up to second order (dipole) allows as to model hadronic effects, significantly improving the precision of the $e-p$ EWC calculations. The calculations are done in the on-shell renormalization scheme using the Feynman gauge. Our theoretical prediction for the PV $e-p$ scattering cross-section asymmetry at $\mathrm{Q}^{2}=0.03 \mathrm{GeV}^{2}$ obtained for the kinematical conditions of the $\mathrm{Q}_{\text {Weak }}$ experiment is $(-0.265 \pm 0.007) \mathrm{ppm}$. The uncertainty comes from the scatter of cuts on the energy of the hard-bremsstrahlung photons. More details are given in [4].

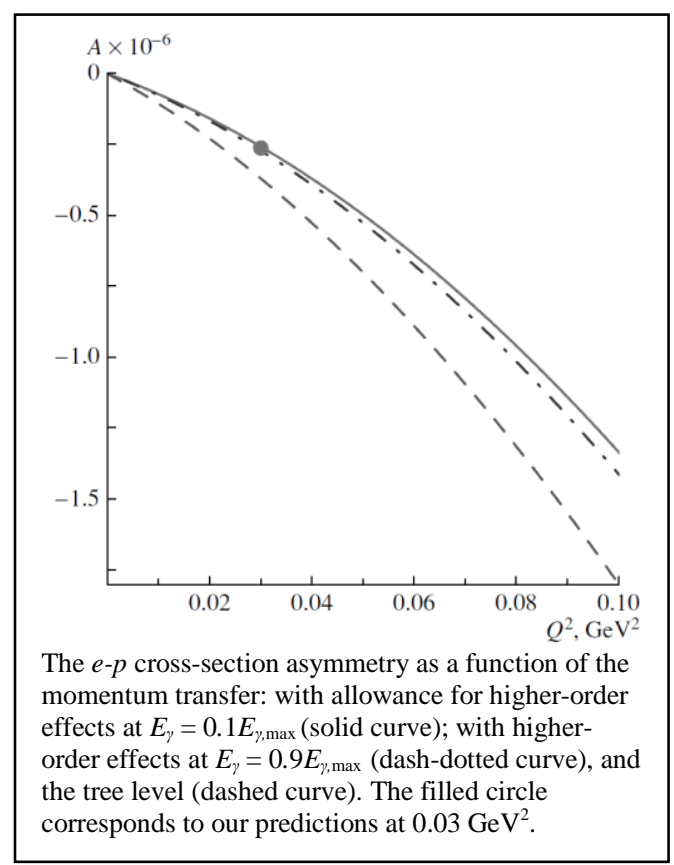

\section{References}

[1] J. Benesch et al., www.jlab.org/ armd/moller_proposal.pdf (2008)

[2] S. Page, JLab E02-020 report to PAC (Qweak-doc-703-v5), at http://www.jlab.org/qweak/ (2007)

[3] A. Aleksejevs et al., Phys. Rev. D 82, 093013 (2010)

[4] A. Aleksejevs et al., Yad. Fiz. 73, 2077 (2010) and Phys. At. Nucl. 73, 2022, (2010) 